\title{
Frustrated Magnetism in Vanadium Oxides
}

\author{
P. Thalmeier, B. Schmidt, V. Yushankhai \\ AND T. TAKImoto \\ Max Planck Institute for Chemical Physics of Solids \\ Nöthnitzer Str. 40, 01187 Dresden, Germany
}

\begin{abstract}
The effect of frustration in various localized and itinerant vanadium oxide compounds is discussed within next nearest neighbors Heisenberg and spin fluctuation models, respectively. In the localized moment case the $S=1 / 2$ $J_{1}-J_{2}$-model on a square lattice exhibits a rich phase diagram with magnetic as well as exotic hidden order phases due to the interplay of frustration and quantum fluctuations. Their signatures in the high field magnetization and in magnetocaloric quantities are surveyed. The possible quantum phase transitions are discussed and applied to layered vanadium oxides of the type $\mathrm{AA}^{\prime} \mathrm{VO}\left(\mathrm{PO}_{4}\right)_{2}$ where $\mathrm{A}, \mathrm{A}^{\prime}=\mathrm{Pb}, \mathrm{Zn}, \mathrm{Sr}, \mathrm{Ba}$, Cd. In itinerant electron systems magnetic frustration may emerge as a result of electron correlations on a geometrically frustrated lattice. This mechanism causes enhanced spin fluctuations in a large region of momentum space and therefore can lead to a heavy fermion state at low temperatures as in the $3 d$ spinel compound $\mathrm{LiV}_{2} \mathrm{O}_{4}$. The evidence from neutron scattering and NMR experiments is discussed within self-consistent renormalization theory based on local density approximation band structure calculations.
\end{abstract}

PACS numbers: 75.10.Jm, 75.10.Lp

\section{Introduction}

In magnetically frustrated compounds the pairwise exchange interactions of spins cannot all be minimized simultaneously in any microscopic moment configuration. This may arise already in the case of nearest neighbor (n.n.) interactions when the lattice has the property of geometric frustration like, e.g. trigonal, Kagomé, checkerboard or pyrochlore type lattices [1-3]. In this case the fundamental building blocks like triangles, plaquettes or tetrahedrons share common sites such that there is no unique state which minimizes all bond energies to nearest neighbor sites. Consequently there will be many states which have approximately equally low energies, i.e., frustration leads to a large degeneracy of low lying states. This entails large quantum fluctuations which may prevent the appearance of magnetic order. Frustration can also arise through the competition of longer range interactions even in simple structures like the $2 \mathrm{D}$ square lattice. At low temperatures there are basically two alternatives: quantum fluctuations may select one of the degenerate states as the true ordered state ("order by disorder") or they may lead to an ordered quantum phase with a new type of order parameter that is of the "hidden order" type, i.e. it does not display a macroscopic modulation of spin density.

The concept and meaning of frustration is much harder to identify in itinerant electron systems. Let us consider non-interacting electrons described by a n.n. tight binding Hamiltonian on a pyrochlore lattice, or its 2D projection, the checkerboard lattice. The kinetic energy alone cannot be "frustrated" since the hopping element $(t)$ is always the same for each n.n. bond. Frustration emerges when one includes electron correlations, e.g. an on-site Coulomb repulsion $U$ or an inter-site repulsion $V$. In the former case and in the limit $U \gg t$ for a half-filled band local moments appear and the exchange energies $\left(J=4 t^{2} / U\right)$ of corner-sharing exchange bonds will be frustrated due to the lattice geometry. This property emerges gradually as $U / t$ increases. For moderate correlations $(U \gtrsim 2 z t, z=$ coordination) and away from half-filling it is better to look at the staggered susceptibility. Geometric frustration may lead to a "flat" dispersion and therefore the interacting susceptibility will be enhanced over a very large region in $\boldsymbol{q}$-space. This can be interpreted as the signature of frustration in an itinerant system. One famous example is the metallic spinel $\mathrm{LiV}_{2} \mathrm{O}_{4}$. The associated softening of spin fluctuation modes in a large part of the Brillouin zone leads to large quasiparticle mass enhancement and therefore to large specific heat $\gamma$-coefficient and susceptibility at low temperatures. Thus geometric frustration may lead to a novel type of $(3 d-)$ heavy fermion state in this compound which is not due to the usual Anderson- or Kondo lattice mechanism.

In this article we first discuss the effects of frustration in the local moment $J_{1}-J_{2}$ model [4-7] on the 2 D square lattice, using both exact diagonalization with finite temperature Lanczos method (FTLM) for finite clusters as well as analytical spin wave methods. In particular, the high field magnetization and magnetocaloric effect in the various phases are investigated (Sect. 2) which are rele- 
vant for a class of layered vanadium oxide compounds. A theory for the itinerant frustrated $3 d$-heavy fermion compound $\mathrm{LiV}_{2} \mathrm{O}_{4}$ [8] will be discussed in Sect. 3. It is based on $a b$ initio local density approximation (LDA) calculations and the self-consistent renormalization (SCR) approach $[9,10]$. It will be used to explain inelastic neutron scattering results which give a direct insight into the origin of frustration in this compound. Finally, Sect. 4 gives a summary.

\section{Frustrated $J_{1}-J_{2}$ magnetism on the square lattice}

The idea of a possible resonating valence band (RVB) state in the cuprates has led to a search for quantum spin liquids in $2 \mathrm{D}$ antiferromagnetic $S=1 / 2$ compounds. In reality most of them exhibit an ordered state with either magnetic or exotic hidden order. In particular the n.n. Heisenberg model on a square lattice has the Néel antiferromagnetic (AF) ground state. A more general case is the $2 \mathrm{D} J_{1}-J_{2}$ model having a control parameter $J_{2} / J_{1}$ which may destabilize the Néel-AF state of the $J_{2}=0$ Heisenberg model. This leads to various other magnetically ordered or hidden order states comprising a rich phase diagram in the $J_{1}-J_{2}$ plane. They also have an interesting behavior in an external field depending on the amount of frustration controled by $J_{2} / J_{1}$. Recently various layered vanadium compounds have been found which correspond well to the $J_{1}-J_{2}$ model. They are of the type $\mathrm{Li}_{2} \mathrm{VOXO}_{4}(\mathrm{X}=\mathrm{Si}, \mathrm{Ge})[11,12]$ and $\mathrm{AA}^{\prime} \mathrm{VO}\left(\mathrm{PO}_{4}\right)_{2}$ $\left(\mathrm{A}, \mathrm{A}^{\prime}=\mathrm{Pb}, \mathrm{Zn}, \mathrm{Sr}, \mathrm{Ba}\right)[13,14]$ and consist of $\mathrm{V}$-oxide pyramid layers containing $\mathrm{V}^{4+}$ ions with $S=1 / 2$. From the analysis of zero field thermodynamic results the frustration ratio $J_{2} / J_{1}$ may be obtained, however an ambiguity remains [4]. A further method of diagnosis is the high field behavior discussed in Sect. 2.2.

\subsection{The $J_{1}-J_{2}$ model and its phases}

The $2 \mathrm{D}$ square lattice $J_{1}-J_{2}$ model in a magnetic field $H$ is given by

$$
\mathcal{H}=J_{1} \sum_{\langle i j\rangle_{1}} \boldsymbol{S}_{i} \cdot \boldsymbol{S}_{j}+J_{2} \sum_{\langle i j\rangle_{2}} \boldsymbol{S}_{i} \cdot \boldsymbol{S}_{j}-h \sum_{i} S_{i}^{z} .
$$

Here $J_{1}$ and $J_{2}$ are two exchange constants between nearest and next nearest neighbors (n.n.n.) on a square lattice, respectively. These parameters are defined per exchange bond and $h=g \mu_{\mathrm{B}} H(g=$ gyromagnetic ratio, $\mu_{\mathrm{B}}=$ Bohr magneton). The phase diagram is preferably characterized by introducing equivalent parameters $J_{\mathrm{c}}=\left(J_{1}^{2}+J_{2}^{2}\right)^{\frac{1}{2}}$ and $\phi=\tan ^{-1}\left(J_{2} / J_{1}\right)$. The angle $\phi$ determines the degree of magnetic frustration.

This model has three possible classical magnetic ground states (see Fig. 1a) depending on $\phi$ : ferromagnet (FM), Néel antiferromagnet (NAF) and collinear antiferromagnet (CAF) [4]. The influence of exchange frustration leading to enhanced quantum fluctuations is strongest at the classical phase boundaries where the $\mathrm{CAF}$ phase joins the $\mathrm{NAF}\left(J_{2} / J_{1}=0.5, \phi=-0.15 \pi\right)$
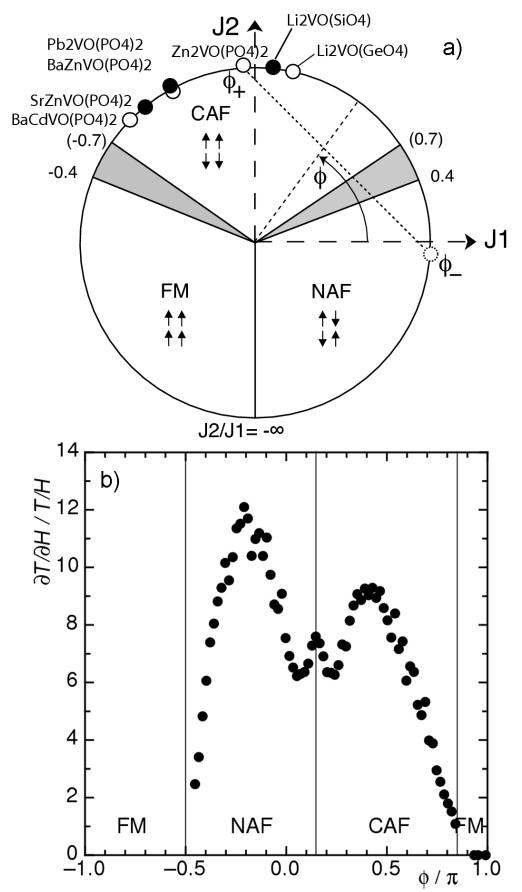

Fig. 1. (a) Phases of the spin-1/2 $2 \mathrm{D}$ square lattice $J_{1}-J_{2}$ model as function of $\phi$ or $J_{2} / J_{1}$. The FM, NAF and CAF order (arrows) have wave vectors $\boldsymbol{Q}=$ $(0,0),(1,1)$ and $(1,0)$ or $(0,1)$ (in units of $\pi / a)$, respectively. The gray sectors $\left(J_{2} / J_{1}\right.$ values indicated outside $)$ represent stacked-dimer phase (right sector) and spin-nematic phase (left sector). Dotted line corresponds to experimental $\Theta_{\mathrm{CW}}=\left(J_{1}+J_{2}\right) / k_{\mathrm{B}}$ for $\mathrm{Zn}$ compound [14] with two possible phases at $\phi_{+}(\mathrm{CAF})$ and $\phi_{-}$(NAF). Full circles correspond to CAF phases determined by neutron scattering [16]. (b) Here $h=h_{\mathrm{s}}(\phi)$ for every $\phi$, i.e. $C_{V}$ and $\Gamma_{\mathrm{mc}}$ are plotted along the curve in the upper inset of Fig. 2, using a fixed $T=0.2 J_{c} / k_{\mathrm{B}}$. The filled circles denote the normalized values $\Gamma_{\mathrm{mc}} /(T / H)$, corresponding to the enhancement relative to a paramagnet (from Ref. [5]).

or $\operatorname{FM}\left(J_{2} / J_{1}=-0.5, \phi=0.85 \pi\right)$ phases. In fact, in these regions they destroy long-range magnetic order [4] and establish two new ordered states, namely a gapped columnar dimer state at the $\mathrm{CAF} / \mathrm{NAF}$ boundary and a gapless spin nematic state at the CAF/FM boundary [6] as shown by the gray sectors in Fig. 1a.

\subsection{Thermodynamics and high field properties of the $J_{1}-J_{2}$ model}

For a compound described by this model it is most important to determine the energy scale $J_{c}$ and especially its frustration parameter $\phi$. For a $J_{1}-J_{2}$ compound the energy scale $J_{c}=\left(J_{1}^{2}+J_{2}^{2}\right)^{\frac{1}{2}}$ is obtained from the high temperature specific heat $C_{V}(T)$ or the temperature $T_{\chi}$ where the susceptibility $\chi(T)$ is at maximum. Furthermore the high-temperature expansion $\left(T \gg J_{c}\right)$ yields the Curie-Weiss constant $\Theta_{\mathrm{CW}}=J_{1}+J_{2}$. We have

$$
\chi(T)=\frac{N_{\mathrm{A}} \mu_{0} g^{2} \mu_{\mathrm{B}}^{2}}{N k_{\mathrm{B}}} \frac{1}{T}\left(\left\langle\left(S_{z}^{\mathrm{tot}}\right)^{2}\right\rangle-\left\langle S_{z}^{\mathrm{tot}}\right\rangle^{2}\right),
$$




$$
C_{V}(T)=\frac{N_{\mathrm{A}}}{N k_{\mathrm{B}}} \frac{1}{T^{2}}\left(\left\langle H^{2}\right\rangle-\langle H\rangle^{2}\right),
$$

here $\langle\ldots\rangle$ is the thermal average, $S_{z}^{\text {tot }}$ is the $z$ component of the total spin of the system, and $N, N_{\mathrm{A}}$ are the number of sites and Avogadro constant respectively, $\mu_{0}$ is the magnetic permeability and $k_{\mathrm{B}}$ - Boltzmann's constant. At zero field $\left\langle S_{z}^{\text {tot }}\right\rangle=0$. Equations (2), (3) are obtained for finite clusters using the FTLM method (they are also valid in finite field). The numerically obtained maximum position $T_{\chi}$ of $\chi(T)$ may be combined with $\Theta_{\mathrm{CW}}=J_{1}+J_{2}$ to the quantity $\Theta_{\mathrm{CW}} / T_{\chi}$ for comparison with experiment. It was shown that no unique solution exists [4] but two possible frustration angles $\phi_{-}$ (NAF region) and $\phi_{+}$(CAF region) are compatible with experimental results for each compound (dotted line in Fig. 1a). One way to distinguish both possibilities is the magnetic structure factor $S(\boldsymbol{q})$ obtained in neutron scattering. From the wave vector of the scattering peak one may decide between $\operatorname{NAF}(\boldsymbol{Q}=(\pi, \pi))$ and $\mathrm{CAF}$ $(\boldsymbol{Q}=(\pi, 0),(0, \pi))$ (Fig. 1a).

\subsection{Magnetization and saturation fields}

Investigation of the uniform magnetization leads to further understanding of the possible ground states of the model [7]. It may be obtained both from numerical Lanczos calculations as well as analytical spin wave expansion starting from the three ordered magnetic phases. In the latter approach a Holstein-Primakoff (HP) transformation leads to a harmonic spin wave Hamiltonian

$$
\mathcal{H}=N E_{0}+N E_{Z P}+\sum_{\boldsymbol{k}} \epsilon_{\boldsymbol{k}}(h) \alpha_{\boldsymbol{k}}^{\dagger} \alpha_{\boldsymbol{k}},
$$

here $\alpha_{\boldsymbol{k}}^{\dagger}$ are magnon operators creating spin waves with dispersion $\epsilon_{\boldsymbol{k}}(h)$. We have, using expressions given in Appendix

$$
\epsilon_{\boldsymbol{k}}(h)=S\left(a_{\boldsymbol{k}}+c_{\boldsymbol{k}}\right)^{\frac{1}{2}}\left(a_{\boldsymbol{k}}+c_{\boldsymbol{k}} \cos \theta_{c}\right)^{\frac{1}{2}},
$$

where $\theta_{c} / 2$ is the field-induced canting angle of sublattice moments (counted from the field direction) with $\theta_{c}=\frac{\pi}{2}$ for $h=0$ and $\theta_{c}=0$ for $h \geq h_{\mathrm{s}}$ where $h_{\mathrm{s}}\left(J_{c}, \phi\right)$ is the saturation field (see upper inset of Fig. 2). For $h<h_{\mathrm{s}}$ minimization of the mean field energy $E_{0}\left(h, \theta_{c}\right)$ leads to the "classical" canting angle $\cos \frac{\theta_{c}}{2}=h / h_{\mathrm{s}}$ with $h_{\mathrm{s}}=8 J_{1} S$ (NAF) and $h_{\mathrm{s}}=S\left(4 J_{1}+8 J_{2}\right)(\mathrm{CAF})$ which results in a linear magnetization $m_{0}=S\left(h / h_{\mathrm{s}}\right)$. In Eq. (4) $E_{\mathrm{ZP}}$ is the energy of zero point fluctuations per site

$$
E_{\mathrm{ZP}}=\frac{1}{2 N} \sum_{\boldsymbol{k}}\left[\epsilon_{\boldsymbol{k}}(h)-S a_{\boldsymbol{k}}\right] .
$$

The zero point energy connected with spin waves will lead to quantum corrections in the magnetization which modify the linear classical behavior according to $m_{\mathrm{ZP}}=$ $-\partial E_{\mathrm{ZP}}(h) / \partial h$ which is determined by the dispersion $\epsilon_{\boldsymbol{k}}(h)$. At the classical phase boundaries CAF/NAF and $\mathrm{CAF} / \mathrm{FM}$ the dispersion becomes anomalous [4]. It is constant (zero for $h \leq h_{\mathrm{s}}$ ) along lines in the Brillouin zone (BZ) connecting the two competing ordering vectors $\boldsymbol{Q}$ at the phase boundary [4]. The expression for the magnetization including quantum corrections up to order
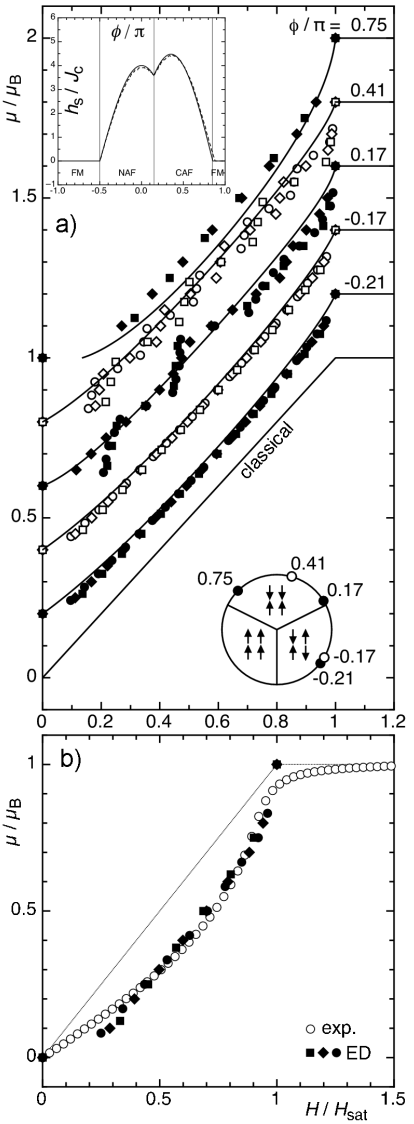

Fig. 2. (a) Magnetization curves $\mu / \mu_{\mathrm{B}}=g m(=m / S)$ for various $\phi$ in the AF or disordered sectors (each curve offset by 0.2). Symbols: $T=0$ Lanczos results for $N=16$ (squares), 20 (diamonds), 24 (dots, circles) size clusters using the Bonner-Fisher construction [15]. Lines: first order spin wave calculations. $\phi / \pi=0.75,-0.21$ correspond to the possible CAF or $\mathrm{NAF}$ values of the $\mathrm{Sr}$ compound. Magnetization curves strongly differ in the extent of nonlinear deviation from the classical curve which corresponds to $\phi / \pi=-0.5$. Deep inside CAF or NAF regions agreement of spin wave and Lanczos calculations is good. The values $\phi / \pi=0.75,0.17$ are nearby or within the nonmagnetic sectors. Close to the CAF/FM boundary the first order spin wave results become unstable at very low fields. At the CAF/NAF boundary the numerical data exhibit a plateau with $m / S=\mu / \mu_{\mathrm{B}}=0.5$. Lower inset shows the position of plotted $\phi$ values in the phase diagram. Upper inset shows the saturation field as function of $\phi\left(h_{\mathrm{s}} \equiv g \mu_{\mathrm{B}} H_{\mathrm{sat}}\right)$ (from Ref. [7]). (b) Nonlinear magnetization curves both from experiments and ED $(\phi / \pi=0.76)$ for the $\mathrm{BaCd}$-compound which is closest to the spin nematic sector in Fig. 1a.

$(1 / S)$ is given by [7]:

$$
m=S \frac{h}{h_{\mathrm{s}}}\left[1-\frac{1}{h_{\mathrm{s}}} \frac{1}{N} \sum_{\boldsymbol{k}} c_{\boldsymbol{k}}\left(\frac{a_{\boldsymbol{k}}+c_{\boldsymbol{k}}}{a_{\boldsymbol{k}}+c_{\boldsymbol{k}} \cos \theta_{c}}\right)^{\frac{1}{2}}\right],
$$

where on the right hand side the classical value of $\theta_{c}$ with $\cos \left(\theta_{c} / 2\right)=h / h_{\mathrm{s}}$ has to be used. Because $h_{\mathrm{s}} \sim S$ the second term in Eq. (7) is formally a $1 / S$ correction to the linear classical term $m_{0}=S\left(h / h_{\mathrm{s}}\right)$. It may be 
expected that these corrections depend on the degree of frustration measured by $\phi$. In the strongly frustrated regime around the classical phase boundaries the dispersion becomes flat along lines in the BZ, thus dramatically increasing the phase space for quantum fluctuations leading to strong nonlinear corrections for the magnetization (Fig. 2). Within the gray sectors magnetic order breaks down and quantum fluctuations stabilize spin nematic (Fig. 2a) [6] and stacked dimer (Fig. 2b) hidden order parameters.

\subsection{Magnetocaloric properties}

Further insight into the quantum phases of the $J_{1}-J_{2}$ model and its high-field behavior may be gained from its magnetocaloric properties [17]. It is a convenient method to determine precisely the saturation field. In practice, the magnetization will be rounded at $h_{\mathrm{s}}$ whereas the magnetocaloric coefficient (the adiabatic cooling rate) has a sharp anomaly from which $h_{\mathrm{s}}$ may be obtained. The magnetocaloric coefficient $\Gamma_{\mathrm{mc}}$ is defined as the rate of adiabatic temperature change with external field

$$
\begin{aligned}
\Gamma_{\mathrm{mc}} & \equiv\left(\frac{\partial T}{\partial H}\right)_{S}=-\frac{T}{C_{V}}\left(\frac{\partial S}{\partial H}\right)_{T} \\
= & -\frac{T}{C_{V}}\left(\frac{\partial m}{\partial T}\right)_{H} .
\end{aligned}
$$

In a paramagnetic system one has $\Gamma_{\mathrm{mc}}^{0}=T / H$ due to the scaling behavior of the free energy. We therefore define $\hat{\Gamma}_{\mathrm{mc}}=\Gamma_{\mathrm{mc}} / \Gamma_{\mathrm{mc}}^{0}$ as the magnetocaloric enhancement due to spin interaction effects. For the $J_{1}-J_{2}$ model $\Gamma_{\mathrm{mc}}$ may be again calculated numerically for finite clusters using the cumulant expression

$$
\Gamma_{\mathrm{mc}} \equiv\left(\frac{\partial T}{\partial H}\right)_{S}=-g \mu_{\mathrm{B}} T \frac{\left\langle\mathcal{H} S_{z}^{\text {tot }}\right\rangle-\langle\mathcal{H}\rangle\left\langle S_{z}^{\text {tot }}\right\rangle}{\left\langle\mathcal{H}^{2}\right\rangle-\langle\mathcal{H}\rangle^{2}}
$$

The magnetocaloric enhancement ratio in FTLM and spin wave approximation (using the last expression in Eq. (8)) exhibit qualitatively similar features: a strong upturn and a positive peak just above the saturation field $h_{\mathrm{s}}$ and for $T \ll J_{c}$ a negative coefficient immediately below $h_{\mathrm{s}}$ [5]. It is instructive to consider the dependence of $\hat{\Gamma}_{\mathrm{mc}}\left(h=h_{\mathrm{s}} ; \phi\right)$ on the frustration angle, keeping the field at saturation level where the maximum of $\Gamma_{\mathrm{mc}}$ occurs. Surprisingly, the maximum enhancement of the magnetocaloric effect (Fig. 1b) occurs in the stable AF regions and not at the classical phase boundaries CAF/NAF and $\mathrm{CAF} / \mathrm{FM}$ where the quantum phases appear due to strong frustration. In these regions a large degeneracy of low lying states (signified by the flat spin wave dispersion along lines in the BZ) should lead to a strong field dependence of the entropy and, according to Eq. (8) to a large $\hat{\Gamma}_{\mathrm{mc}}$.

However, let us note that the specific heat $C_{V}$ occurs in the denominator of Eq. (8). It also shows a strong enhancement in the quantum phase regions $(\phi \approx 0.15 \pi$, $\phi \approx 0.85 \pi$ ), this overcompensates the increase in the numerator in Eq. (8). Therefore, the magnetocaloric enhancement $\hat{\Gamma}_{\mathrm{mc}}$ is only moderate in these regions while its maxima occur in the middle of the NAF or CAF phase sectors in Fig. 1b. The measurement of $\Gamma_{\mathrm{mc}}(h)$ should be an excellent method to determine the saturation field $h_{\mathrm{s}}$ in the $J_{1}-J_{2}$ compounds. Their absolute values for the known layered $\mathrm{V}$-oxides are ranged between 5 and $25 \mathrm{~T}[5]$.

\section{Itinerant frustrated heavy fermion compound $\mathrm{LiV}_{2} \mathrm{O}_{4}$}

The metallic spinel compound $\mathrm{LiV}_{2} \mathrm{O}_{4}$ is the first $3 d$ heavy electron system discovered [8]. Below $30 \mathrm{~K}$ a large specific heat and the Pauli susceptibility enhancement appears, achieving $\gamma=C / T=0.4 \mathrm{~J} /\left(\mathrm{mol} \mathrm{K}{ }^{2}\right)$ for the former at the lowest temperatures. Many proposals to explain this behavior have been made, including traditional Kondo-like scenarios. A special feature of the spinels and therefore of $\mathrm{LiV}_{2} \mathrm{O}_{4}$ is the fact that $\mathrm{V}$ atoms reside on a pyrochlore lattice, their average electron count is $n_{d}=1.5$ per $\mathrm{V}$. In a simple tight binding picture this corresponds to quarter-filling (in the hole picture) of $d$-bands, i.e. the system is far from the localized Mott limit. The exchange-correlation effects, however, still exist which may lead to the development of pronounced short range spin correlations in the paramagnetic metallic state of $\mathrm{LiV}_{2} \mathrm{O}_{4}$. This approach was developed in Ref. [9] within random phase approximation (RPA) spin fluctuation theory based on $a b$ initio LDA electronic structure.

\subsection{Electronic structure and spin susceptibility}

The pyrochlore lattice has four atoms per unit cell. A next neighbor tight binding model for a single orbital [18] leads to four bands where the upper twofold degenerate band is completely flat. The real band structure of $\mathrm{LiV}_{2} \mathrm{O}_{4}$ keeps a resemblance to this simple model with a large DOS peak on top. However, the Fermi level is far below the top and this peak does not directly play a role for the enhanced $\gamma$-value. Nevertheless, the flat upper band is indirectly important within the spin fluctuation mechanism of mass enhancement. For this model one needs to calculate the dynamic and momentum dependent spin susceptibility.

The result of this calculation [9] for various subcritical exchange strengths is shown in Fig. 3 for the [111]-direction in momentum space. Together with results for [100] and [110] it shows that surprisingly the susceptibility is enhanced by approximately the same factor in a nearly spherical region with a radius $Q_{c} \approx 0.6 \AA^{-1}$ and a finite thickness $\delta Q \approx 0.45 \AA^{-1}$ in momentum space. This is the signature of frustration for itinerant spin fluctuations. Since the static $\chi(\boldsymbol{Q}) \sim \chi\left(Q_{c}\right)$ is almost degenerate in this shell it means that the system, although close to a magnetic instability, has no obvious way to select an ordering wave vector. As a consequence, the dynamical susceptibility will show a slowing down (shifting the spectral function weight to very low energies) in the whole critical shell in the BZ. Therefore, there is a large phase space of low energy spin fluctuations which 

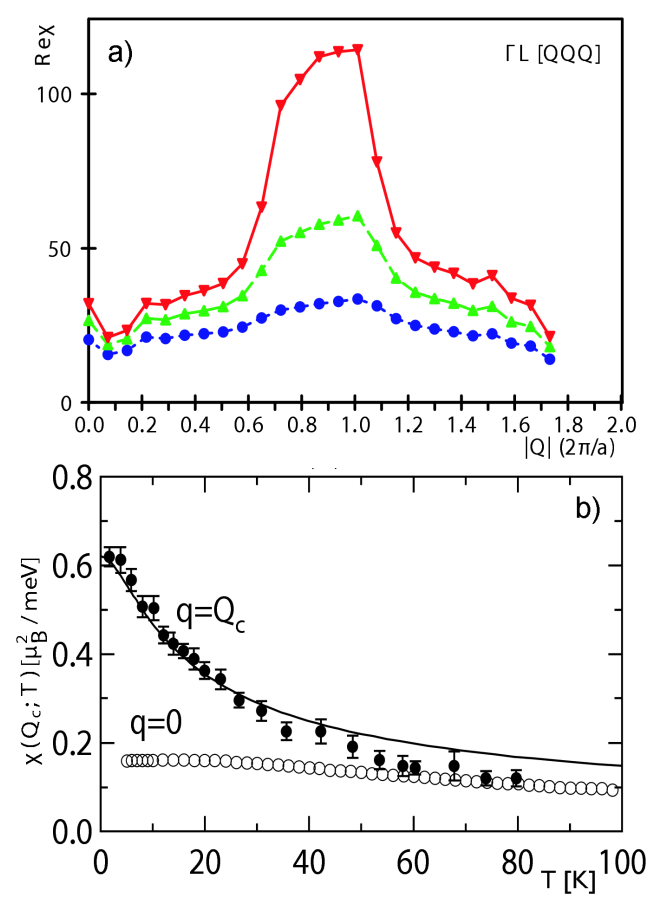

Fig. 3. (a) Static spin susceptibility $\chi(\boldsymbol{Q}, 0)$ along [111] direction for different values of local exchange coupling constant $K<K_{c}(K=0.45,0.40,0.30$ from top to bottom). The critical exchange coupling is $K_{c}=0.49 \mathrm{eV}$ and $2 \pi / a \approx 0.76 \AA^{-1}$ (from Ref. [9]). (b) Static susceptibilities at $q=Q_{c}$ (full circles) and $q=0$ (open circles) as functions of temperature observed in INS and magnetic measurements on $\mathrm{LiV}_{2} \mathrm{O}_{4}$, respectively. The solid line is a fit to $\chi\left(Q_{c}, T\right)$ using the self-consistent solution of $y_{Q_{c}}(T)$ (from Ref. [10]).

can renormalize the quasiparticle mass. This situation is quite different from non-frustrated lattices where the enhancement of the interacting susceptibility is usually sharply peaked around the incipient magnetic ordering vector, providing only a small phase space and moderate quasiparticle mass enhancement through spin fluctuations.

\subsection{Mass enhancement and inelastic neutron scattering}

When $\boldsymbol{Q}$ is located within the critical shell the dynamical susceptibility describing low energy spin fluctuations may be approximately written as $(Q=|\boldsymbol{Q}|)$

$\operatorname{Im} \chi(Q, \omega) \simeq z_{Q} \chi(Q) \omega / \Gamma(Q)$,

where $\Gamma(Q)$ and $z_{Q}<1$ are their energy width and weight, respectively. Since $\chi(Q)$ is much enhanced and $\Gamma(Q)$ small in the critical shell the spectral function around $Q_{c}$ is strongly peaked at low energies in agreement with inelastic neutron scattering (INS) results [19-21]. The conduction electrons are dressed with these low energy bosonic excitations leading to a large spin fluctuation specific heat $\gamma_{s f}=C_{s f} / T$ below $60 \mathrm{~K}$ as given by

$$
\gamma_{s f}=\frac{k_{\mathrm{B}}^{2} \pi}{N} \sum_{\boldsymbol{q}} \frac{z(\boldsymbol{q})}{\hbar \Gamma(\boldsymbol{q})} .
$$

Since $\Gamma(\boldsymbol{q})$ is small in the whole critical shell around $|\boldsymbol{q}|=Q_{c}$ this may lead to a large $\gamma_{s f}$. The absolute scale of the spin fluctuation width, $\Gamma$ is estimated to fall between the limits $0.5 \mathrm{eV}<\Gamma<1.5 \mathrm{meV}$ with a corresponding $300>\gamma_{s f}>100$ in units of $\mathrm{mJ} /\left(\mathrm{mol} \mathrm{K}^{2}\right)$. This result shows that slow spin fluctuations over extended momentum region, caused by the effect of frustration, may explain the size of the large $\gamma$ value in $\mathrm{LiV}_{2} \mathrm{O}_{4}$ and its heavy-fermion character.

\subsection{Mode-mode coupling effects}

At larger temperatures the spin fluctuation modes of different $\boldsymbol{q}$ couple with each other leading to a transfer of spectral weight from the critical region to low momentum region. Experimentally, it was observed that above $60 \mathrm{~K}$ the susceptibility enhancement in the critical shell around $q=Q_{c}$ vanishes and becomes equal to the value at $q=0$. The effect of mode coupling on the static susceptibility may be described within Moriya's SCR theory [22] according to

$$
\begin{aligned}
& \chi(\boldsymbol{q}, T)^{-1}=\chi(\boldsymbol{q}, 0)^{-1} \\
& \quad+\bar{F}_{Q_{c}} \int_{0}^{\infty} \frac{\mathrm{d} \omega}{2 \pi} \frac{1}{\mathrm{e}^{\omega / T}-1} \frac{1}{N} \sum_{\boldsymbol{q}^{\prime}} \operatorname{Im} \chi\left(\boldsymbol{q}^{\prime}, \omega, T\right) .
\end{aligned}
$$

Here $\bar{F}_{Q_{c}}$ is a mode--mode coupling constant for the critical shell. The reduced inverse susceptibility may be written as $y\left(Q_{c}, T\right)=\frac{1}{2 T_{\mathrm{A}} \chi_{Q_{c}}(T)}$ where $T_{\mathrm{A}} \approx 220 \mathrm{~K}$ is a scale parameter. It is obtained from numerical solution of the SCR integral equation derived from Eq. (12) [23]. The resulting temperature dependence of the critical susceptibility $\chi_{Q_{c}}(T)$ together with corresponding experimental results and those of $q=0$ are shown in Fig. 3b. It clearly demonstrates that the critical enhancement of $\chi_{Q_{c}}(T)$ is rapidly reduced with temperature and approaches that of the FM point $q=0$. Since the ratio of scattering intensities at $q=Q_{c}$ and $q=0$ is proportional to the square of the susceptibilities, Fig. 3b implies that the critical scattering intensity at $Q_{c}$ is reduced by almost a factor 16 when temperature increases to $60 \mathrm{~K}$. Recently it was shown [23] that SCR theory can also explain the $T$ - and p-dependence of NMR relaxation rate in $\mathrm{LiV}_{2} \mathrm{O}_{4}$.

\section{Summary}

A class of layered vanadium oxides are realizations of the $2 \mathrm{D} J_{1}-J_{2}$ model. They are all lying in the CAF sector of the phase diagram (with the possible exception of the Zn-compound). Of particular interest are those close to the spin nematic hidden order phase like the $\mathrm{BaCd}$ -compound (Fig. 1a). In this case anomalous nonlinear magnetization due to pronounced quantum fluctuations appear indicating the instability of magnetic order and closeness to nonmagnetic (spin nematic) order (Fig. 2). The enhancement of magnetocaloric cooling rate is a useful method to determine the saturation field. It is largest within the stable magnetic sectors (Fig. 1b). 
It has been proposed that the intriguing $3 d$-heavy fermion compound $\mathrm{LiV}_{2} \mathrm{O}_{4}$ is a frustrated metal close to a magnetic instability. This explains naturally the momentum and temperature dependence of neutron scattering results (Fig. 3b). The associated slowing down of low lying spin fluctuations in conjunction with the large instability region in $\boldsymbol{q}$-space due to frustration effects was found to explain the quasiparticle mass enhancement.

\section{Appendix}

The sublattice coupling energies $a_{\boldsymbol{k}}, b_{\boldsymbol{k}}$ and $c_{\boldsymbol{k}}$ appearing in Sect. 2.3 are given by

$$
\begin{gathered}
(\mathrm{NAF}): \quad a_{\boldsymbol{k}}=4\left[J_{1}-J_{2}\left(1-\bar{\gamma}_{\boldsymbol{k}}\right)\right], \\
c_{\boldsymbol{k}}=-b_{\boldsymbol{k}}=4 J_{1} \gamma_{\boldsymbol{k}}, \\
(\mathrm{CAF}): \quad a_{\boldsymbol{k}}=2\left(2 J_{2}+J_{1} \gamma_{y}\right), \\
c_{\boldsymbol{k}}=-b_{\boldsymbol{k}}=2\left(J_{1}+2 J_{2} \gamma_{y}\right) \gamma_{x}
\end{gathered}
$$

for the two AF phases. Here the momentum structure factors are defined by $\gamma_{\boldsymbol{k}}=\frac{1}{2}\left(\cos k_{x}+\cos k_{y}\right), \bar{\gamma}_{\boldsymbol{k}}=$ $\cos k_{x} \cos k_{y}, \gamma_{x}=\cos k_{x}$ and $\gamma_{y}=\cos k_{y}$.

\section{Acknowledgments}

The authors would like to thank P. Fulde, N. Shannon, A. Yaresko, and M. Zhitomirsky for collaboration and discussions.

\section{References}

[1] G. Misguich, C. Lhuillier, in: Frustrated Spin Systems, Ed. H.T. Diep, World Sci., Singapore 2004.

[2] P. Fulde, P. Thalmeier, G. Zwicknagl, Solid State Physics, Vol. 60, Amsterdam 2006, p. 1.

[3] G. Misguich, P. Sindzingre, Eur. Phys J. B 59, 305 (2007).

[4] N. Shannon, B. Schmidt, K. Penc, P. Thalmeier, Eur. Phys. J. B 38, 599 (2004).

[5] B. Schmidt, P. Thalmeier, N. Shannon, Phys. Rev. B 76, 125113 (2007).
[6] N. Shannon, T. Momoi, P. Sindzingre, Phys. Rev. Lett. 96, 027213 (2006).

[7] P. Thalmeier, M. Zhitomirsky, B. Schmidt, N. Shannon, Phys. Rev. B 77, 104441 (2008).

[8] S. Kondo, C. Johnston, C.A. Swenson, F. Borsa, A.V. Mahajan, L.L. Miller, T. Gu, A.I. Goldman, M.B. Maple, D.A. Gajewski, E.J. Freeman, N.R. Dilley, R.P. Dickey, J. Mermin, K. Kojima, G.M. Luke, Y.J. Uemara, O. Chmaissem, J.D. Jorgensen, Phys. Rev. Lett. 78, 3729 (1997).

[9] V. Yushankhai, A. Yaresko, P. Fulde, P. Thalmeier, Phys. Rev. B 76, 085111 (2007).

[10] V. Yushankhai, P. Thalmeier, T. Takimoto, Phys. Rev. B 77, 094438 (2008).

[11] P. Millet, C. Satto, Mater. Res. Bull. 33, 1339 (1998).

[12] R. Melzi, S. Aldrovandi, F. Teboldi, P. Carretta, P. Millet, F. Mila, Phys. Rev. B 64, 024409 (2001).

[13] E.E. Kaul, H. Rosner, N. Shannon, R.V. Shpanchenko, C. Geibel, J. Magn. Magn. Mater. 272-276, 922 (2004).

[14] N. Kini, E.E. Kaul, C. Geibel, J. Phys., Condens. Matter 18, 1303 (2006).

[15] J.C. Bonner, M.S. Fisher, Phys. Rev. 135, A640 (1964).

[16] M. Skoulatos, J.P. Goff, N. Shannon, E. Kaul, C. Geibel, A.P. Murani, M. Enderle, A.R. Wildes, J. Magn. Magn. Mater. 310, 1257 (2007).

[17] M.E. Zhitomirsky, Phys. Rev. B 67, 104421 (2003).

[18] M. Isoda, S. Mori, J. Phys. Soc. Jpn. 69, 1509 (2000).

[19] A. Krimmel, A. Loidl, M. Klemm, S. Horn, H. Schober, Phys. Rev. Lett. 82, 2919 (1999).

[20] S.H. Lee, Y. Qiu, C. Broholm, Y. Ueda, J.J. Rush, Phys. Rev. Lett. 86, 5554 (2001).

[21] A. Murani, A. Krimmel, J. Stewart, M. Smith, P. Strobel, A. Loidl, A. Ibarra-Palos, J. Phys., Condens. Matter 16, S607 (2004).

[22] T. Moriya, Spin Fluctuations in Itinerant Electron Magnetism, Springer Ser. Solid-State Science, Vol. 56, Springer, Berlin 1985.

[23] V. Yushankhai, T. Takimoto, P. Thalmeier, J. Phys. Condens. Matter 20, 465221 (2008). 